\title{
El cambio de la enseñanza y el aprendizaje en tiempos de pandemia
}

\author{
Rafael Porlán \\ Didáctica de la Ciencias Experimentales, Departamento de Didáctica de las Ciencias \\ Experimentales y Sociales, Facultad de Educación, Universidad de Sevilla.rporlan@us.es \\ ORCID: https://orcid.org/0000-0003-2068-7092
}

[Recibido: 14 Junio 2020. Revisado: 14 Junio 2020. Aceptado: 14 Junio 2020]

\begin{abstract}
Resumen: Ante la crisis producida por el coronavirus se ha puesto en evidencia la fragilidad de la sanidad pública y el deterioro sufrido en los últimos años. De la misma manera ha ocurrido con la educación. En todos los niveles educativos, docentes, familias, estudiantes y responsables académicos han tenido que responder de manera urgente a una situación nueva: la imposible presencialidad. Este hecho inevitable ha puesto en evidencia también profundas carencias del sistema educativo y diferentes alternativas para afrontarlos. En este breve escrito comentaremos los aspectos que nos parecen esenciales para afrontar la escuela y la universidad que vienen, entre ellos el hecho de que los recursos tecnológicos por sí mismos no producen un cambio docente, aunque pueden ayudar a promoverlo
\end{abstract}

Palabras Claves: Modelo de educación. Enseñanza presencial. Enseñanza no presencial. Recursos tecnológicos.

The change in teaching and learning in times of pandemic

Abstract: Faced with the crisis caused by the coronavirus, the fragility of public health and the deterioration suffered in recent years has become evident. The same has happened with education. At all educational levels, teachers, families, students and academic leaders have had to respond urgently to a new situation: the impossible attendance. This inevitable fact has also exposed deep deficiencies in the educational system and different alternatives to face them. In this brief writing we will comment on the aspects that seem essential to us to face the coming school and university, among which the technological resources, although they are not the change, but can help to promote it. Without forgetting that the changes are in the people and not in the resources.

Key Words: Education model. Face-to-face teaching. Non-classroom teaching. Technological resources.

Para citar este artículo: Porlán, R. (2020) El cambio de la enseñanza y el aprendizaje en tiempos de pandemia. Revista de Educación Ambiental y Sostenibilidad 2(1), 1502. doi: 10.25267/Rev_educ_ambient_sostenibilidad.2020.v2.i1.1502

Los momentos de crisis producen escenarios para nuevas tomas de decisiones y ponen en evidencia problemas que permanecían ocultos a ojos de la mayor parte de la ciudadanía. El ejemplo más importante que estamos viviendo en estos momentos es el relativo a la sanidad pública. Ante la crisis producida por el coronavirus, se ha puesto en evidencia su fragilidad y el deterioro sufrido en los últimos tiempos debido a las privatizaciones y recortes. Es posible que muchos ciudadanos estén tomando conciencia ahora de que el valor de la sanidad pública es algo que va más allá de las diferencias políticas, y que debería ser un elemento transversal que todos deberíamos cuidar. Algo similar puede estar ocurriendo, aunque probablemente en menor grado, con la evidencia de que la pandemia tiene una relación estrecha con el tipo de relación que como especie mantenemos con los ecosistemas y la biosfera. También en este caso, se abren oportunidades para una mayor conciencia ambientalista en el conjunto de la población. 
De la misma manera ha ocurrido con la educación. En todos los niveles educativos, docentes, familias, estudiantes y responsables académicos han tenido que responder de manera urgente a una situación nueva: la imposible presencialidad en los centros educativos. Este hecho inevitable ha puesto en evidencia también profundas carencias del sistema educativo y diferentes alternativas para afrontarlos. En este breve escrito comentaremos los aspectos que nos parecen esenciales para afrontar la escuela y la universidad que vienen.

\section{El modelo de enseñanza-aprendizaje vigente no es adecuado ni para la educación presencial ni para la no presencial}

No es una cuestión de gustos. Es una cuestión de rigor y profesionalidad. Con lo que hoy sabemos desde la Neurociencia, la Comunicación Humana, las Ciencias de la Educación, la Psicología y Sociología del Aprendizaje, etc. no es sostenible una enseñanza basada esencialmente en la transmisión directa del saber desde el docente al estudiante, se haga en un espacio físico o virtual. Y las razones son las siguientes:

a) Se aprende con la mente en on y no con la mente en off. Es decir, para aprender de manera profunda es imprescindible la actividad mental del sujeto y esto no suele ocurrir en el papel de consumidor pasivo de información. Con la mente en off el sujeto obedece y simula o desobedece e incomoda, pero no aprende, solo en on está potencialmente capacitado para aprender.

b) Lo que se propone aprender ha de ser relevante. La relevancia puede responder a diferentes razones que pueden variar según el nivel educativo: Las cuestiones que se abordan conectan al sujeto con problemáticas reales y atractivas de cierto grado de importancia (social, cultural, ambiental, profesional, personal, etc.); La manera cómo se plantean interroga, activa y cuestiona las ideas y estereotipos previos del sujeto; el que aprende no las percibe como algo acabado y cerrado sino como un reto en el que puede implicarse...

c) Lo que se propone aprender ha de ser organizador. No es posible abordarlo todo ni todo es igual de importante. Hay asuntos que tienen un carácter organizador, tanto en las disciplinas como en los esquemas mentales personales. Una visión sistémica de la realidad, por ejemplo, en la que las interacciones, observables o no, son la clave, es un contenido organizador porque permite un cambio cualitativo en la visión del mundo. La enseñanza ha de ir donde el estudiante solo no llega. Un aprendizaje profundo es incompatible con un temario enciclopédico e indiscriminado porque abordar los asuntos organizadores requiere tiempo, implicación y sosiego.

d) Lo que se propone aprender no puede estar fragmentado. El saber, en su sustrato físico, químico y biológico (neuronal), es conexión y reacción. Es imposible aprender nada de manera aislada. La comprensión está en la interacción entre significados. Las ideas aisladas mueren. Los sistemas de ideas permanecen, se desarrollan y se reconstruyen en cada nueva interacción.

e) La manera de aprender es investigativa y constructiva. Nadie puede sustituir al que aprende. Dar el contenido puede tranquilizar porque guarda la apariencia del deber cumplido y exime de toda responsabilidad. Pero el saber no se puede dar, no es un ente trasplantable de una mente a otra. El saber es construido por cada 
mente de manera idiosincrática. La investigación es una cualidad común a todo ser humano, que permite plantearse problemas, formular hipótesis desde los saberes previos, contrastarlas y construir nuevos saberes. El desarrollo o la inhibición de esta capacidad es una de las grandes responsabilidades del sistema educativo. La ciencia es el resultado de un desarrollo de alto nivel de esta capacidad y de su cultivo en contextos profesionalizados, pero no es algo privilegiado de algunas personas, en su base es un atributo común de la especie.

f) La manera de ayudar a aprender es poner andamios en el proceso. El sujeto investiga y construye y el docente le pone los andamios para el proceso. Aunque incompleta, esta metáfora es muy visual. La ayuda (la enseñanza) es indirecta pero imprescindible. Empezamos por la base, lo que el sujeto es capaz de hacer por sus propios medios y con sus propios saberes para abordar problemas, proyectos, retos o casos que estén a su alcance, el resultado será parcial e imperfecto, con errores, con visiones insuficientes sobre el fenómeno. Esa es su base. Para seguir el docente pone los andamios para el primer piso, los mejores andamios posibles para su construcción (ahí está la complejidad de la enseñanza), pero el que construye es el sujeto y lo que construye es su saber. Así hasta construir su particular edificio cognitivo.

g) El proceso de aprender es individual y social y está impregnado de emociones. Somos una especie social y aprendemos y construimos en sociedad. También somos seres profundamente emocionales y esto nos impulsa o nos bloquea el aprendizaje. Y ambas cuestiones están interrelacionadas, porque gran parte de nuestra emoción y de nuestra autoestima se construye en la interacción social. Reconocer y tratar al que aprende como persona capaz es la primera piedra del edificio emocional y cognitivo. Dar el contenido, desde la mejor voluntad, tiene el efecto indeseado de anular al que aprende. Liberar y alimentar un proceso colectivo de aprendizaje humaniza a todos los participantes: al que enseña (que también aprende) y a los que aprenden (que también enseñan). Es un escenario de profunda humanidad y de democracia real. Y eso es emocionante. Y la emoción amplifica la capacidad mental de conexión, interacción y construcción de saber. Un impresionante proceso de retroalimentación circular.

h) La evaluación como un proceso de ajuste continuo entre la enseñanza y el aprendizaje. La evaluación centrada en el control y la cuantificación del saber reproducido (apariencia de saber) estimula inconscientemente la simulación, y la negación y el ocultamiento del saber propio. El que aprende no dice lo que cree sino lo que cree que tiene que decir. Esto tiene muy pocos beneficios para el auténtico aprendizaje pues inhabilita al docente a ajustar su enseñanza a las necesidades emergentes en el proceso de construcción real del que aprende. La evaluación, por tanto, no debería ser otra cosa que la investigación rigurosa del docente sobre qué y cómo evoluciona el aprendizaje real para, por un lado, poder mejorar y afinar su intervención y, por otro, aportar información valiosa al que aprende para su propia autorregulación. Pero también al contrario, una evaluación completa recorre el camino inverso cuando el que aprende manifiesta sus necesidades y sus opiniones sobre la intervención y actuación del docente, ayudando a mejorar sus propuestas de enseñanza y su labor de andamiaje. Por tanto, se necesita una evaluación investigativa, formativa y democrática orientada 
hacia la mejora del proceso y hacia el mejor ajuste posible entre la enseñanza y el aprendizaje.

\section{Los recursos tecnológicos no son el cambio, pero pueden ayudar a promoverlo}

Desmontado el modelo de enseñanza-aprendizaje vigente, puesto en evidencia en muchos momentos durante la pandemia, irrumpe de manera impetuosa, al hilo de la no presencialidad, una verdad revelada: es necesario un profundo cambio basado en las nuevas tecnologías del aprendizaje y la comunicación. El problema central, se argumenta, es que la escuela y la universidad no están preparadas para el uso de los nuevos recursos tecnológicos. Es necesaria una revolución digital para poner la educación a la altura de los tiempos. Una verdad parcial no es una verdad completa. Nadie puede negar que el uso del teléfono incrementó en su momento las posibilidades de comunicación humana, pero ¿cambió el contenido de las conversaciones? Es importante hilar fino ante este tipo de deslumbramientos tecnológicos. En México he tenido la oportunidad de conocer experiencias innovadoras en territorios indígenas, con un alto contenido transformador, en línea con el modelo descrito en el apartado anterior, y con una tecnología de bajo perfil. Aporto aquí, por tanto, algunos de los criterios a tener en cuenta ante esta revelación:

a) Los modelos mentales se cambian por otros modelos mentales, pero no por el hecho en sí de usar nuevas herramientas tecnológicas. La enseñanza transmisiva, por más que se considere la manera natural de enseñar, se corresponde con un determinado modelo mental (normalmente interiorizado de forma inconsciente), y la incorporación de cualquier medio o recurso se pone al servicio del mismo, de manera que, por ejemplo, de dar el temario presencialmente podemos pasar a darlo virtualmente sin que nada cambie en el fondo, aunque cambie la forma.

b) Los autores de estas herramientas también tienen un modelo, consciente o no, sobre la enseñanza y el aprendizaje, que lógicamente suele ser el vigente, y los recursos que diseñan suelen ser coherentes con él. Prueba de ello es que los más utilizados y ofertados durante la pandemia tienen que ver con la posibilidad de dar clases magistrales en formato virtual (controlando la asistencia), de subir apuntes o power point para el estudio de los alumnos y para acabar el temario, de grabar vídeos expositivos, de realizar exámenes virtuales a prueba de la copia entre alumnos, etc., provocando con todo ello la frustración de muchas familias y estudiantes que no solo no han visto ninguna revolución educativa, sino un más de lo mismo empobrecido por la distancia física y emocional, en momentos de crisis humana y social.

c) Los recursos tecnológicos, la innovación tecnológica y el propio concepto de innovación es un potente nicho de mercado en el ámbito educativo. No es inocente que grandes multinacionales como Movistar, Santander, La Caixa, Santillana, etc, inviertan tanto dinero en promover un determinado modelo de innovación educativa (que podríamos denominar neoliberal y tecnológico) vinculado a plataformas digitales promocionadas por dichas compañías. Por tanto, seamos conscientes de que la educación es un objeto de mercado y de consumo, y vinculemos el uso de nuevas herramientas tecnológicas a los movimientos y 
experiencias que promueven recursos de libre disposición para apoyar modelos sociales transformadores.

d) Junto a todo lo anterior, también ha habido en este tiempo experiencias que responden a otro modelo de escuela y universidad y que han utilizado con otro sentido todo tipo de medios tecnológicos para promover el aprendizaje investigativo y constructivo. He tenido la oportunidad de participar directamente en el Proyecto del colectivo Con+Ciencia titulado Investigando en casa, donde docentes de primaria, universidad y estudiantes del grado de Educación Primaria hemos diseñado $\mathrm{y}$ experimentado juntos, diversas unidades didácticas investigativas sobre problemas como el uso de los plásticos en casa, el modo de vida de los abuelos, el funcionamiento de las máquinas cotidianas, las historias de vida de la familia, etc., con una participación extraordinaria de familias y estudiantes y con unos resultados realmente esperanzadores. He de añadir, que el recurso más utilizado ha sido el móvil y diferentes aplicaciones de uso libre que han facilitado la comunicación e interacción entre los participantes. Pueden conocerse las unidades ya experimentadas, junto a otras no relacionadas con este proyecto concreto, en el siguiente enlace: https://sites.google.com/view/conmascienciarecursos/home/con-ciencia-en-casa

\section{La relación humana directa y presencial es insustituible}

Junto al deslumbramiento tecnológico, que olvida que los cambios están en las personas y no en los recursos, aunque, insisto, éstos pueden facilitarlos, ha eclosionado también con mucha fuerza la idea de la educación a distancia. Conviene también aquí detenerse a analizar los matices de esta alternativa, pues, como se ha venido observando durante la pandemia, su implantación extraordinaria ha venido acompañada de múltiples instrucciones y adaptaciones normativas, pero ninguna relativa a la correspondiente regulación laboral del trabajo que implica para los docentes. Podrían los responsables políticos caer en la tentación de sobrevalorar interesadamente este tipo de enseñanza, pues implica menor coste que una reducción de la ratio y una contratación adecuada de profesorado para la escuela y la universidad que viene. Estas son algunas de las reflexiones acerca de la necesidad de la presencialidad:

a) Las relaciones humanas que se establecen en el contacto directo son más ricas y complejas que las que se producen a distancia. Como sabemos, el cerebro humano procesa mucha más información de la que le llega por trasmisión verbal, siendo la comunicación no verbal (gestual, auditiva, corporal, etc.) una parte esencial del diálogo entre las personas. Las pantallas limitan en exceso dicha comunicación y condicionan la espontaneidad y el flujo de sensaciones, predominando la frialdad y la distancia frente al acercamiento, la empatía y el acompañamiento. Como hemos visto anteriormente, las emociones son el sustrato de la relación didáctica, y tienen la capacidad de acelerar o bloquear los procesos de aprendizaje. Salvo casos excepcionales, es difícil sostener, por nuestra propia experiencia, que la interacción a distancia puede cubrir las mismas necesidades emocionales que el contacto directo. La vuelta de los seres queridos es el mejor ejemplo que tenemos.

b) Hemos afirmado que el aprendizaje es un fenómeno social y lo es en diversas dimensiones: La sociedad nos influye; La familia, la pareja y los amigos nos 
enseñan; La escuela es una pequeña sociedad; etc. Pero nos interesa poner el acento en el trabajo cooperativo dentro del aula. El debate entre iguales (bien orientado), en relación a los asuntos que se estén investigando tiene un valor educativo fundamental: desarrolla la capacidad de argumentación, promueve el contraste de puntos de vista, ayuda a los estudiantes con niveles de elaboración más bajos a avanzar en su edificio cognitivo (pues los compañeros hacen un andamiaje intuitivo), promueven la autorregulación emocional y constituyen un auténtico sistema inteligente que supera a la suma de las partes. Argumentos similares podríamos utilizar en relación al funcionamiento del grupo-clase y a las interacciones con los docentes. Todo esto es francamente muy difícil de promover en la educación a distancia.

c) La llamada modalidad dual (parte presencial y parte no presencial) es, en cierta medida, algo que algunos docentes ya venimos practicando. En mis clases, por ejemplo, las tareas y proyectos que se llevan a cabo para resolver los retos y problemas que centran el aprendizaje, se desarrollan en el tiempo de clase y se continúan fuera de clase. Lo que en el modelo transmisivo es el estudio para los exámenes, en otros casos se convierte en la continuidad del proceso investigativo, con tareas en las que nuestra presencia directa no es tan necesaria. Las diferentes metodologías que podrían situarse dentro del modelo constructivista e investigativo (Aprendizaje Basado en Problemas o en Proyectos, la Clase Invertida -en su versión más avanzada-, el Estudio de Casos, etc. ) suelen dar continuidad al trabajo presencial en el ámbito no presencial Por tanto, desde este modelo alternativo, las tareas no presenciales son algo consustancial. Sin embargo, desde la perspectiva del modelo vigente, que es desde la que se hace la propuesta, la dualidad significa que unas clases (transmisivas) se dan presencialmente y otras (también transmisivas) no se dan presencialmente. El comentario ya está dicho desde el principio: el modelo transmisivo no es adecuado, en cualquiera de los dos formatos, y si se propone es más por un problema de costes económicos que de planteamiento pedagógico.

En resumen, la escuela y la universidad necesitan un cambio profundo en el modelo de enseñanza y aprendizaje dominante y la pandemia lo ha puesto en evidencia. Es muy descorazonador leer los innumerables twits de los estudiantes de la Universidad de Sevilla en \#QuemadUS (o en otras cuentas en la mayoría de las universidades). Copio solo dos ejemplos: "Cuando los docentes están más preocupados por evaluar que por enseñar, sus alumnos se interesan más por aprobar que por aprender"; "Universidad de Sevilla: ¿Amas la profesión, empatizarías con los alumnos e impartirías tu asignatura de manera que el estudiantado trabaje de forma sana? Respuesta: No; Universidad de Sevilla: Perfecto, estás admitido".

El cambio necesario, por tanto, no es un asunto de presencialidad o no presencialidad sino es un profundo cambio de mentalidad, que requiere de procesos de formación en la acción del profesorado, en todos los niveles del sistema educativo, y de una auténtica transformación de la formación inicial (incorporándola, por fin, al caso de la universidad). También de cambios estructurales imprescindibles como la disminución de la ratio, la contratación de nuevo profesorado, una legislación que permita la transición entre modelos, etc. 
La revolución que se necesita, por tanto, es la de adecuar el Modelo de Enseñanza a lo que científicamente sabemos sobre el aprendizaje, y desde ahí cobra sentido el uso adecuado de determinadas, y muy útiles, herramientas tecnológicas. 